\title{
ELABORACIÓN Y VALIDACIÓN DE UNA REGLA DE PREDICCIÓN CLÍNICA PARA IDENTIFICAR COMPROMISO SISTÉMICO EN CASOS DE LOXOSCELISMO
}

\author{
PREPARATION AND VALIDATION OF A SYSTEMIC LOXOSCELISM PREDICTION PROTOCOL
}

Rafael Pichardo-Rodriguez', Marcos Saavedra-Velasco², Jhonatan Ascarza-Saldaña', Cesar Naquira-Velarde³

\begin{abstract}
RESUMEN
Introducción: El loxoscelismo sistémico es la complicación más severa del loxoscelismo. El manejo del cuadro por parte del personal de salud presenta una alta variabilidad por factores que se desconocen actualmente. No se cuenta con un estándar de referencia ni con un modelo de predicción clínica que pueda guiar nuestras decisiones al momento del abordaje de un paciente con mordedura de araña. Objetivo: Elaborar y validar una regla de predicción clínica para loxoscelismo sistémico. Métodos: Se llevó a cabo un estudio observacional de derivación y validación de un modelo de predicción clínica con validación de prueba diagnóstica basada en una cohorte histórica de un solo brazo en pacientes atendidos en el Hospital Vitarte entre los años 2007 al 2016 y reportes clínicos internacionales publicados. Resultados: El loxoscelismo sistémico se presentó solo en el 32,9\% $(n=24)$ de casos. Para el análisis bivariado, las variables que demostraron presentar una asociación estadísticamente significativa $(P<0,05)$ fueron el sexo, mordedura en abdomen independiente en relación a otras partes del cuerpo, mordedura en otras partes del cuerpo que no sea el abdomen, vómito, fiebre y hemoglobinuria. El análisis de regresión incluyó en el análisis a las variables: sexo, vómito, fiebre y hemoglobinuria. El bootstrapping determinó la validez interna del modelo. El área bajo la curva fue de $0,91(\mathrm{P}<0,05)$ y la sensibilidad, espeficidad, LR+ y LR-fueron de 79,1\%, 93,8\%, 12,9 y 0,22 respectivamente. Conclusión: El protocolo de predicción del loxoscelismo sistémico derivado es válido, por el momento.
\end{abstract}

Palabras clave: Venenos de arañas; Araña reclusa parda; Predicción (fuente: DeCS BIREME).

\begin{abstract}
Introduction: Systemic loxoscelism is the most severe complication of loxoscelism. The management of the cadre by health personnel presents a high variability due to factors that are currently unknown. There is no standard of reference or a clinical prediction model that can guide our decisions when approaching a spider bite patient. Objective: Develop and validate a clinical prediction rule for systemic loxoscelism. Methods: An observational study of derivation and validation of a clinical prediction model was carried out with diagnostic test validation based on a historical single-arm cohort in patients treated at Vitarte Hospital between 2007 and 2016 and international clinical reports published. Results: Systemic loxoscelism occurred only in $32.9 \%(n=24)$ of cases. For the bivariate analysis, the variables that showed a statistically significant association $(\mathrm{P}<0.05)$ were sex, bite in an independent abdomen in relation to other parts of the body, bite in other parts of the body than the abdomen, vomiting, fever and hemoglobinuria. The regression analysis included in the analysis the variables: sex, vomit, fever and hemoglobinuria. Bootstrapping determined the internal validity of the model. The area under the curve was $0.91(P<0.05)$ and the sensitivity, specificity, LR + and LR- were $79.1 \%, 93.8 \%, 12.9$ and 0.22 respectively. Conclusion: The protocol of prediction of systemic derived loxoscelism is valid, for the moment.
\end{abstract}

Key words: Spider venoms; Brown recluse spider; Prediction (source: MeSH NLM).

\footnotetext{
${ }^{1}$ Clínica de Urología Avanzada UROZEN, Lima-Perú.

${ }^{2}$ Hospital Nacional Edgardo Rebagliati Martins, Lima-Perú.

${ }^{3}$ Instituto de Investigación en Ciencias Biomédicas. Universidad Ricardo Palma, Lima-Perú.

Citar como: Rafael Pichardo-Rodriguez, Marcos Saavedra-Velasco, Jhonatan Ascarza-Saldaña, Jhony A De La Cruz-Vargas, Cesar Naquira-Velarde. Elaboración y validación de una regla de predicción clínica para identificar compromiso sistémico en casos de loxoscelismo. Rev. Fac. Med. Hum. Octubre 2020; 20(1):32-42. DOI 10.25176/RFMH.v20i1.2642

Artículo publicado por la Revista de la Facultad de Medicina Humana de la Universidad Ricardo Palma. Es un artículo de acceso abierto, distribuído bajo los términos de la Licencia Creative Commons: Creative Commons Attribution 4.0 International, CC BY 4.0 (https://creativecommons.org/licenses/by/4.0/), que permite el uso no comercial, distribución y reproducción en cualquier medio, siempre que la obra original sea debidamente citada. Para uso comercial, por favor póngase en contacto con revista.medicina@urp.pe
} 


\section{INTRODUCCIÓN}

El diagnóstico y predicción del cuadro sistémico de loxoscelismo continúa siendo un misterio y un reto para el clínico que maneja a los pacientes con mordedura de araña. No hay un estándar de referencia para el diagnóstico, pese a las investigaciones y desarrollo de pruebas rápidas de inmunoensayos (ELISA). Estas pruebas rápidas de ELISA han mostrado resultados exitosos en países europeos, pese a ser desarrolladas en base a especies de Loxosceles norteamericanas, sin embargo, no están validadas para diferentes poblaciones y no están disponibles en todos los países ${ }^{(1-3)}$. Así mismo, no se ha podido establecer hasta la fecha una predicción de la evolución del envenenamiento, que es un punto clave para el manejo y reducción de la mortalidad, morbilidad y costes para el paciente y para el presupuesto nacional de salud. Pese a encontrarse variables o factores asociados a loxoscelismo sistémico como presentar una mordedura a nivel del tórax, fiebre y mal estado general dentro de las primeras 24 horas; como lo reporta Webb et $\mathrm{al}^{(4)}$, no hay una evaluación de su capacidad diagnóstica ni predictiva.

Además, se presenta gran variabilidad en el diagnóstico y tratamiento entre los médicos, realidad que se observa tanto a nivel nacional como internacional, debido a que no se cuenta con procedimientos estandarizados, ni con una guía de práctica clínica. Motivo por el que el objetivo del presente estudio fue elaborar y validar una regla de predicción clínica para identificar casos de compromiso sistémico en pacientes atendidos por loxoscelismo.

\section{MÉTODOS}

\section{Diseño, lugar y tiempo de estudio}

Se llevó a cabo un estudio observacional de derivación y validación de una regla de predicción clínica basada en una cohorte de un solo brazo en pacientes atendidos en el Hospital Vitarte entre los años 2007 al 2016 y reportes clínicos internacionales publicados.

Por ser una patología de baja frecuencia, se replicó el método de reclutamiento de dos orígenes de pacientes aplicado por Xian Han et al ${ }^{(5)}$ en un estudio prospectivo de una neoplasia hematológica muy infrecuente. Unos de los orígenes de pacientes son aquellos que son atendidos en un hospital y otros aquellos presentados en publicaciones científicas (reportes de caso).

\section{Criterios de selección}

Se incluyeron a aquellos pacientes atendidos en el Hospital Vitarte entre los años 2007 al 2016 con diagnóstico confirmado de loxoscelismo (cutáneo y sistémico) y aquel reporte de caso publicado en bases de datos internacionales (web of science, scopus, pubmed y scielo) y que presentaron las variables de estudio (mordedura en tórax, abdomen, inicio de fiebre, naúseas, vómitos y mal estado general en las primeras 24 horas de evolución, ictericia, hemoglobinuria, niveles elevados de bilirrubina total, creatinina y LDH) posterior a la mordedura. Se excluyeron a aquellos pacientes con historia clínica extraviada e incompleta y aquellos que presentaron ántrax cutáneo, mordedura de araña de otro género, picadura de insectos (abeja africana y/o Lonomia sp), herpes simple infectado crónico, úlcera diabética, necrólisis epidérmica tóxica y eritema nodoso multiforme.

\section{Definición de enfermedad, variables y puntos finales}

Se definió como loxoscelismo sistémico a la mordedura de una araña del género loxosceles asociado a la afectación de un órgano diferente a la piel (anemia hemolítica, falla renal, injuria hepática aguda, entre otros). Adicionalmente se estudiaron las siguientes variables: Sexo, edad, mordedura en tórax, abdomen, inicio de fiebre, naúseas, vómitos y mal estado general en las primeras 24 horas de evolución, ictericia, hemoglobinuria, niveles elevados de bilirrubina total, creatinina y LDH.

\section{Métodos y procedimientos para la medición y recolección de las variables}

Los datos fueron registrados a partir de las historias clínicas de los pacientes en fichas de datos elaboradas en base a los objetivos de estudio.

Tiempo de seguimiento: Los participantes fueron seguidos y controlados desde el momento de la mordedura, hasta 5 días posteriores al accidente.

a) Recolección de datos de publicaciones internacionales:

Se colocaron los descriptores de ciencias de la salud (DeCS) y "Medical Subject Headings" (MeSH): Loxoscelismo, loxoscelism, loxosceles, loxosceles Reclusa, Case Reports, reporte de caso, serie de caso, series case, Spider Venom, Brown Recluse Spider. En las diferentes bases de datos internacionales: PUBMED, Web of Science, Ovid PubMed, LILACS, LIPECS, SCIELO y Open Grey. Se incluyeron aquellos reportes y series de caso donde el accidente fue compatible con la 
clínica del loxoscelismo, confirmándose el diagnóstico y/o se identificó la araña. Se recolectaron los datos en la ficha de datos en base a los criterios de selección.

b) Recolección de datos de pacientes atendidos en el hospital Vitarte:

Se envió una solicitud de aceptación y ejecución del proyecto de tesis a la oficina de apoyo a la docencia e investigación (OADI) del Hospital Vitarte, posterior a la aprobación del proyecto por parte del Consejo de Facultad y el Instituto de Investigación en Ciencias Biomédicas (INICIB) de la Facultad de Medicina Humana "Manuel Huamán Guerrero" de la Universidad Ricardo Palma.

Posterior a la aprobación se solicitó el número de casos de loxoscelismo refiriendo el código CIE-10 de veneno de araña: T63.3, al servicio de estadística, obteniendo el número de pacientes y su número de historia clínica desde el año 2007 hasta el 2016 con accidente por arácnido. Se ubicó cada historia clínica y se seleccionaron a los que cumplieron con los criterios de selección, recolectando la información necesaria en la ficha de datos.

\section{Cálculo del tamaño de muestra}

Para un nivel de confianza del $95 \%$, con una proporción esperada de casos de loxoscelismo con compromiso sistémico del $5 \%$ y una precisión de $5 \%$ se requirió un total de 73 pacientes para completar el estudio. La selección de la muestra fue por conveniencia, incluyendo todos los casos encontrados.

\section{Aspectos éticos}

No se experimentó en seres humanos. Se guardó y protegió la confidencialidad de los datos, todo participante tuvo derecho al anonimato, respetándose así los principios de las buenas prácticas clínicas propuestos en la declaración de Helsinki.

\section{Análisis estadísticos}

Se realizó un análisis estadístico descriptivo con presentación de porcentaje y frecuencias para las variables cualitativas, y medias, desviación estándar o mediana y rango para las cuantitativas en base a los resultados de las pruebas de normalidad usando Shaphiro-Wilk. Posteriormente se realizó el análisis bivariado evaluando la asociación entre posibles predictores (se consideró estadísticamente significativo a un $\mathrm{p}<0,05)$. Para la asociación estadística entre variables cualitativas con más de dos categorías, se realizó la prueba Post Hoc de Bonferroni al Chi cuadrado de Pearson, considerándose estadísticamente significativo un valor de $p<0,05$.
Aquellas variables con un valor de " $p$ " menor a 0,05 fueron incluidos en el modelo de regresión logística binaria por el método "STEPwise". A las variables seleccionadas por el modelo de regresión de nuestro protocolo de predicción, se presentaron sus respectivos Odds Ratio (OR) con su respectivo intervalo de confianza al 95\% (IC-95\%) y el valor de p. La capacidad discriminativa del protocolo se evaluó mediante el análisis de curvas operativas del receptor (ROC). Los puntos de corte para el protocolo fueron obtenidos en base a la determinación de likelihood ratio (LR) positivo (LR+) y negativo (LR-) y el índice de Youden. Se calcularon: sensibilidad, especificidad para el mejor punto de corte. Para la determinación de la validez interna del modelo se realizó un re-muestreo con 50 repeticiones por medio de la técnica Bootstrapping, presentando sus respectivos coeficientes de origen, arranque y sesgo. Cada coeficiente representó un puntaje específico para cada variable constituyendo el protocolo de predicción, presentando para cada puntaje, así como a cada síntoma o sumatoria de ellos su respectiva probabilidad para el desarrollo de loxoscelismo sistémico. Se elaboró un nomograma de Fagan basado en el LR+y las probabilidades pre-test y post-test a partir de la simulación de tres escenarios clínicos diferentes en las que se aplicó el protocolo de predicción (Escenario 1 [Línea Roja] un clínico presenta una probabilidad pre-prueba del $10 \%$ en un paciente que acude a su consulta por mordedura de araña. Escenario 2 [Línea verde] el mismo clínico presenta una probabilidad pre-prueba del $50 \%$. Escenario 3 [Línea azul] el mismo clínico presenta una probabilidad pre-prueba del $80 \%$ en un paciente que presenta otra mordedura de araña). Los datos fueron procesados y analizados en el software estadístico STATA Versión 14 (Stata Corp. Texas, U.S.).

\section{RESULTADOS}

Se incluyeron para el análisis a 73 pacientes, de los cuales 49 provinieron de reportes y series de caso (Tabla 1) y 25 del hospital Vitarte. Del total, el loxoscelismo sistémico se presentó en el 32,9\% $(n=24)$ de casos, de los cuales 21 casos provinieron de los reportes y series de caso y 3 casos del hospital Vitarte. Las características clínicas de los pacientes con loxoscelismo se presentan en la Tabla 2.

Las variables accidente en época de verano, en otras épocas del año, náuseas, mal estado general, nivel bajo de hemoglobina, nivel elevado de bilirrubina indirecta, creatinina, LDH, prolongación del tiempo de protrombina y complicaciones presentaron datos perdidos en más del $50 \%$ haciendo imposible su utilización. 
Para el análisis bivariado, las variables que demostraron presentar una asociación estadísticamente significativa con el desarrollo del cuadro sistémico del loxoscelismo fueron la edad, sexo, mordedura en otras partes del cuerpo que no es el abdomen, vómito, fiebre y hemoglobinuria. Las variables mordedura en tórax, palidez e ictericia presentaron dentro de la tabla de contingencia el valor de 0 , el cual hace imposible el cálculo de los análisis estadísticos bivariados, motivo por el que no se utilizó los resultados para los análisis estadísticos siguientes. La prueba de Post Hoc de Bonferroni para el Chi Cuadrado de Pearson dio un resultado estadísticamente significativo $(P=0,02)$ para mordedura en abdomen en comparación a las diferentes categorías. Ver resultados en la Tabla 1.

El análisis de regresión incluyó a las variables: sexo, vómito, fiebre y hemoglobinuria. No se tomaron a las variables edad, mordedura en abdomen ni mordedura en otras partes del cuerpo que no es el abdomen. En la Tabla 2 se resumen los resultados.

El modelo de predicción mostró un área bajo la curva de 0,91 $(\mathrm{P}<0,05)$, con un índice de Youden de 0,73 y criterio de clasificación o punto de corte de 0,35 para el valor de predicción calculado por el modelo siendo equivalente a un puntaje de 4 en la regla de predicción derivada. La sensibilidad fue de 79,1\% y la especificidad de 93,8\%, el LR+ fue de 12,9 y LR- de 0,22 . En la Figura 1 se observa la curva ROC del modelo predictivo.

El bootstrapping para la determinación de la validez interna del modelo para 50 repeticiones mostró coeficientes de origen y de arranque de 0,68 y 0,69 (IC-95\%: 0,68-0,70) y el sesgo solo fue de 0,008 (IC95\%: 0,007-0,009), corroborando la validez interna del modelo.

La regla de predicción clínica derivada con sus respectivos puntajes se muestra en la Tabla 3.

Los resultados de los puntajes del protocolo derivado son:

- 2 puntos: $14 \%$ de probabilidad de presentar loxoscelismo sistémico
- 3 puntos: $22 \%$ de probabilidad de presentar loxoscelismo sistémico

- 4 puntos: 57\% de probabilidad de presentar loxoscelismo sistémico

- 9 puntos: 98\% de probabilidad de presentar loxoscelismo sistémico

- 11 puntos: 99\% de probabilidad de presentar loxoscelismo sistémico

Al combinar las probabilidades de los síntomas presentados por los pacientes obtenemos:

- Sexo masculino: $14 \%$ de probabilidad de presentar loxoscelismo sistémico.

- Fiebre: $22 \%$ de probabilidad de presentar loxoscelismo sistémico.

- Vómito: $14 \%$ de probabilidad de presentar loxoscelismo sistémico.

- Hemoglobinuria: 57\% de probabilidad de presentar loxoscelismo sistémico.

- Sexo masculino + fiebre: $62 \%$ de probabilidad de presentar loxoscelismo sistémico.

- Sexo masculino + fiebre + hemoglobinuria: $98 \%$ de probabilidad de presentar loxoscelismo sistémico.

- Sexo masculino + fiebre + vómito + hemoglobinuria: 99\% de probabilidad de presentar loxoscelismo sistémico.

En la Figura 2, se presenta el diagrama de Fagan que muestra tres escenarios clínicos: Escenario 1 (Línea Roja) un clínico presenta una probabilidad pre-prueba del $10 \%$ en un paciente que acude a su consulta por mordedura de araña y al aplicar el modelo predictivo presenta una probabilidad post-test de $64 \%$ de padecer loxoscelismo sistémico. Escenario 2 (Línea verde) el mismo clínico presenta una probabilidad preprueba del $50 \%$ en un paciente con otra mordedura de araña, la probabilidad post-test sube al 95\%. Escenario 3 (Línea azul) el mismo clínico presenta una probabilidad pre-test de $80 \%$ en un paciente que presenta otra mordedura de araña, al aplicar el modelo predictivo la probabilidad post-test sube a un $99 \%$. 
Tabla 2. Características clínicas de los pacientes con loxoscelismo.

\begin{tabular}{|c|c|c|c|}
\hline & Loxoscelismo sistémico (n=24) & Loxoscelismo cutáneo ( $n=49)$ & P valor \\
\hline Edad & 11 años (Rango 0,3-92) & 28 años (Rango 1-80) & $P=0,020$ \\
\hline \multicolumn{4}{|l|}{ Sexo } \\
\hline Femenino & $6(25 \%)$ & $30(61,2 \%)$ & \multirow[t]{2}{*}{$P=0,040$} \\
\hline Masculino & $18(75 \%)$ & $19(38,8 \%)$ & \\
\hline \multicolumn{4}{|c|}{ Tiempo de enfermedad } \\
\hline Menor de 24 horas & $9(37,5 \%)$ & $25(51 \%)$ & \multirow[t]{2}{*}{$P=0,270$} \\
\hline Mayor de 24 horas & $15(62,5 \%)$ & $24(49 \%)$ & \\
\hline \multicolumn{4}{|l|}{ Lugar de lesión } \\
\hline Abdomen & $5(20,8 \%)$ & $2(4,1 \%)$ & \multirow[t]{11}{*}{$P=0,080$} \\
\hline Cara & $1(4,2 \%)$ & $7(14,3 \%)$ & \\
\hline Cuello & $2(8,3 \%)$ & $1(2 \%)$ & \\
\hline Dorso & $1(4,2 \%)$ & $0(0 \%)$ & \\
\hline Escroto & $0(0 \%)$ & $1(2 \%)$ & \\
\hline Mama & $0(0 \%)$ & $1(2 \%)$ & \\
\hline Miembro inferior & $6(25 \%)$ & $19(28,8 \%)$ & \\
\hline Miembro superior & $7(29,2 \%)$ & $11(22,4 \%)$ & \\
\hline Oreja & $1(4,2 \%)$ & $2(4,1 \%)$ & \\
\hline Parpado & $0(0 \%)$ & $5(10,2 \%)$ & \\
\hline Tórax & $1(4,2 \%)$ & $0(\%)$ & \\
\hline \multicolumn{4}{|l|}{ Mordedura en Tórax } \\
\hline $\mathrm{Si}$ & $1(4,2 \%)$ & $0(0 \%)$ & \multirow[t]{2}{*}{ No calculable* } \\
\hline No & $23(95,8 \%)$ & $0(0 \%)$ & \\
\hline \multicolumn{4}{|c|}{ Mordedura en Abdomen** } \\
\hline $\mathrm{Si}$ & $5(20,8 \%)$ & $2(4,1 \%)$ & \multirow[t]{2}{*}{$\mathrm{P}=0,020$} \\
\hline No & $19(79,2 \%)$ & $47(95,9 \%)$ & \\
\hline \multicolumn{4}{|c|}{ Mordedura en otras partes del cuerpo que no es el abdomen } \\
\hline $\mathrm{Si}$ & $18(75 \%)$ & $47(95,9 \%)$ & \multirow[t]{2}{*}{$P<0,007$} \\
\hline No & $6(25 \%)$ & $2(4,1 \%)$ & \\
\hline \multicolumn{4}{|l|}{ Vómito } \\
\hline $\mathrm{Si}$ & $12(50 \%)$ & $2(4,1 \%)$ & \multirow[t]{2}{*}{$P=0,001$} \\
\hline No & $12(50 \%)$ & $47(95,9 \%)$ & \\
\hline \multicolumn{4}{|l|}{ Fiebre } \\
\hline $\mathrm{Si}$ & $16(66,7 \%)$ & $6(12,2 \%)$ & \multirow[t]{2}{*}{$P=0,001$} \\
\hline No & $8(33,3 \%)$ & $43(87,8 \%)$ & \\
\hline \multicolumn{4}{|l|}{ Palidez } \\
\hline Si & $15(62,5 \%)$ & $0(0 \%)$ & \multirow[t]{2}{*}{ No calculable } \\
\hline No & $9(37,5 \%)$ & 49 (100\%) & \\
\hline \multicolumn{4}{|l|}{ Ictericia } \\
\hline $\mathrm{Si}$ & $10(41,7 \%)$ & $0(0 \%)$ & \multirow[t]{2}{*}{ No calculable } \\
\hline No & $14(58,3 \%)$ & 49 (100\%) & \\
\hline \multicolumn{4}{|l|}{ Hemoglobinuria } \\
\hline $\mathrm{Si}$ & $12(50 \%)$ & $1(2 \%)$ & \multirow[t]{2}{*}{$P=0,001$} \\
\hline No & 12 (50\%) & 48 (98\%) & \\
\hline \multicolumn{4}{|l|}{ Muerte } \\
\hline $\mathrm{Si}$ & $4(15 \%)$ & $2(4,1 \%)$ & \multirow[t]{2}{*}{$P=0,087$} \\
\hline No & $20(75 \%)$ & $47(95,9 \%)$ & \\
\hline
\end{tabular}

*Dentro de la tabla de contingencia se presenta el 0 que hace incalculable los análisis bivariados.

** Resultado estadísticamente significativo de la prueba de Post Hoc de Bonferroni para el Chi Cuadrado de Pearson.

Pág. 36 
Tabla 1. Reportes y series de casos incluidas en el estudio.

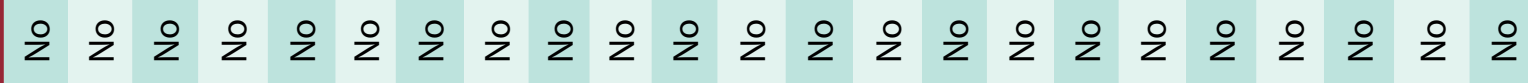

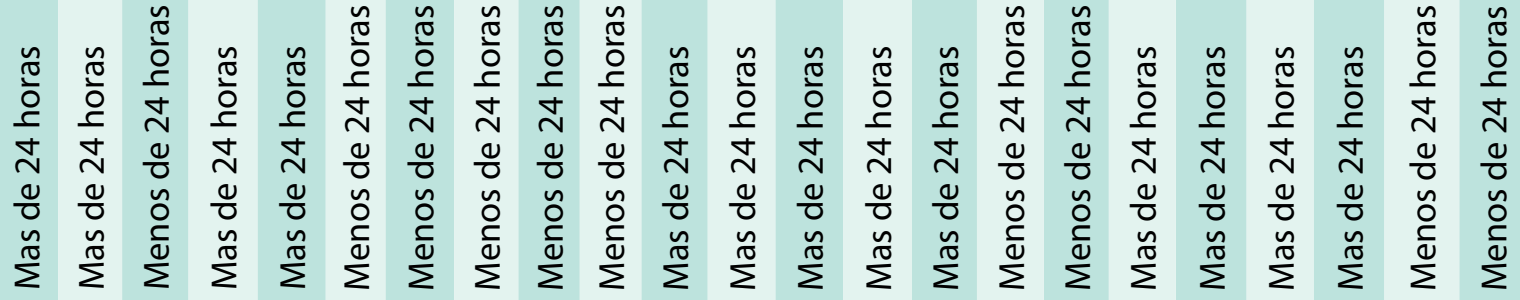

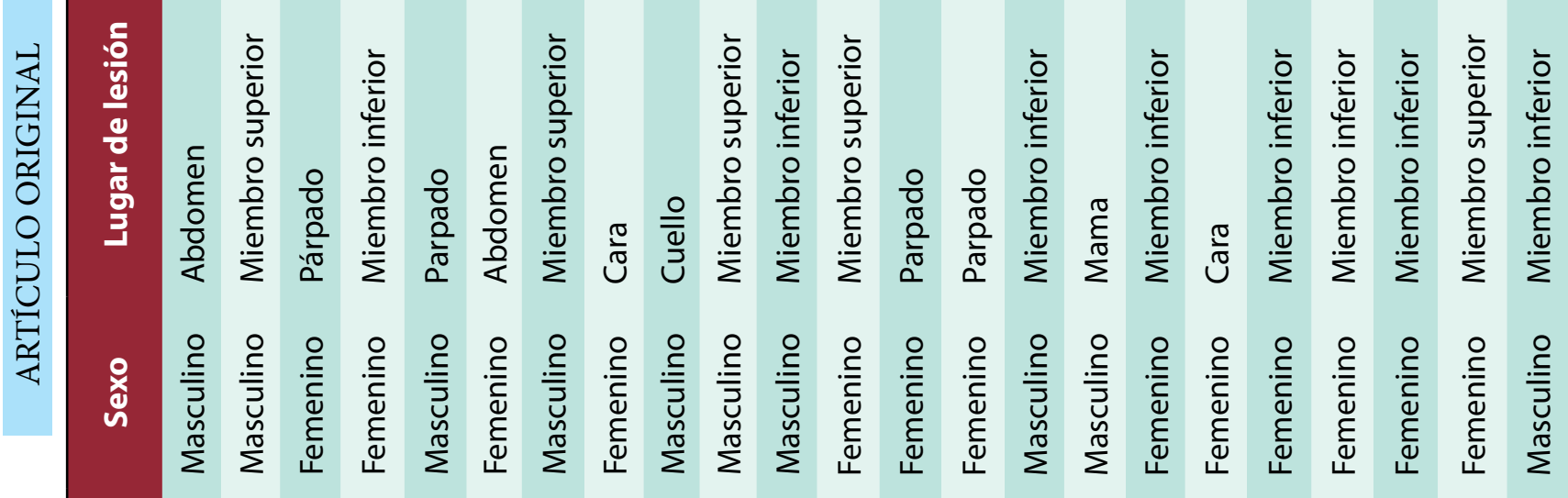

胥

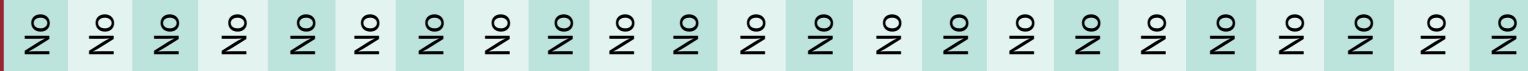

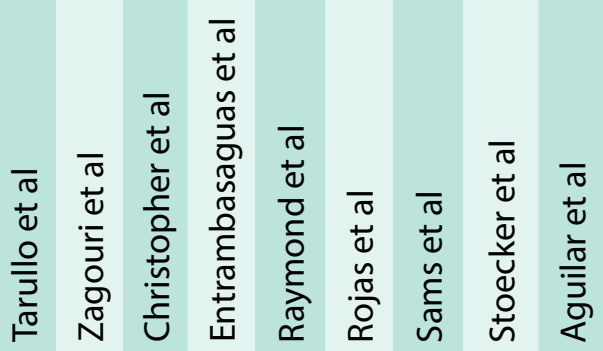

을

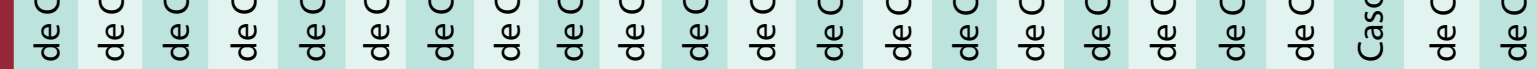

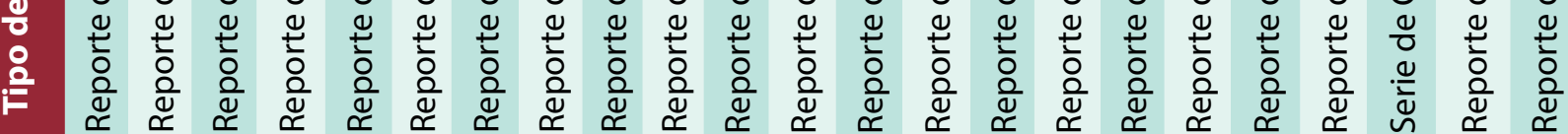




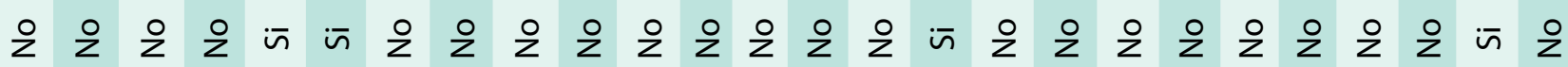

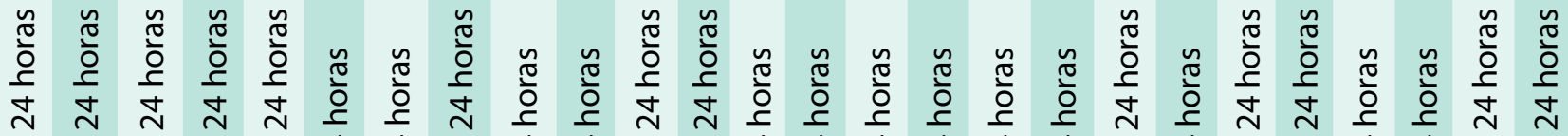

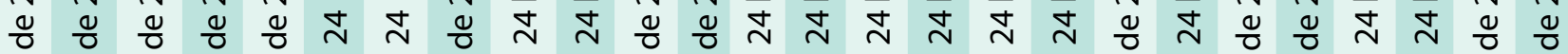
彳ิ 乞ั

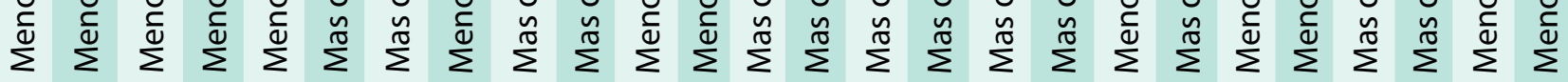

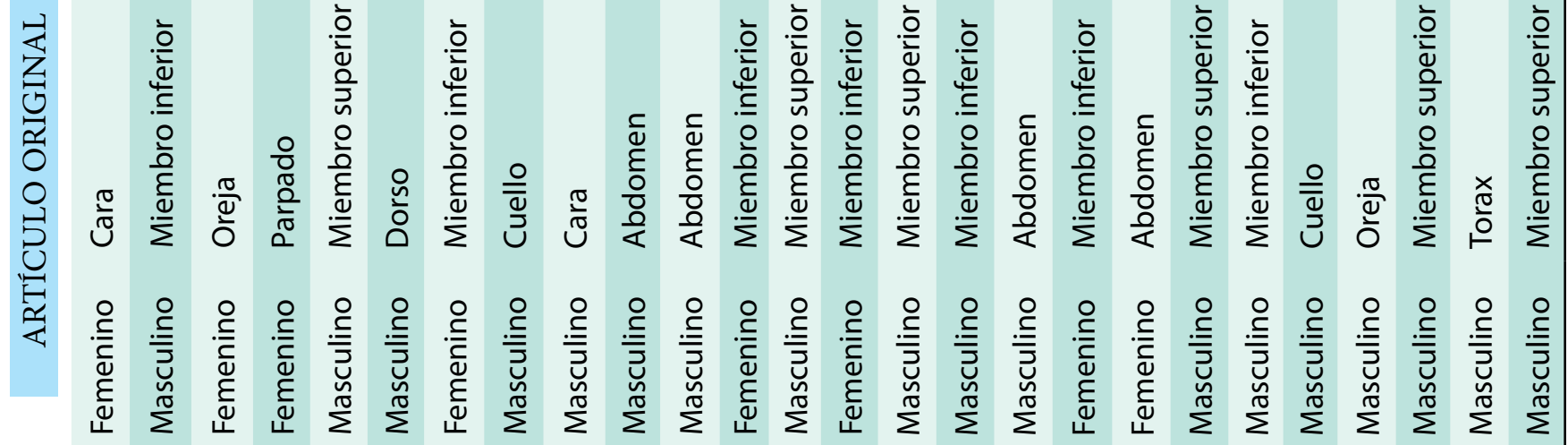
$\infty \stackrel{m}{m}$ ำ

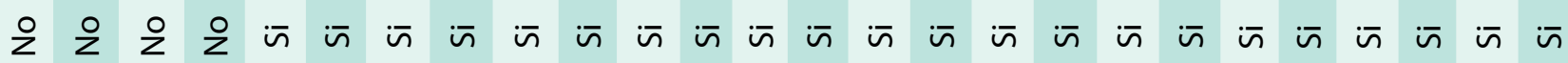

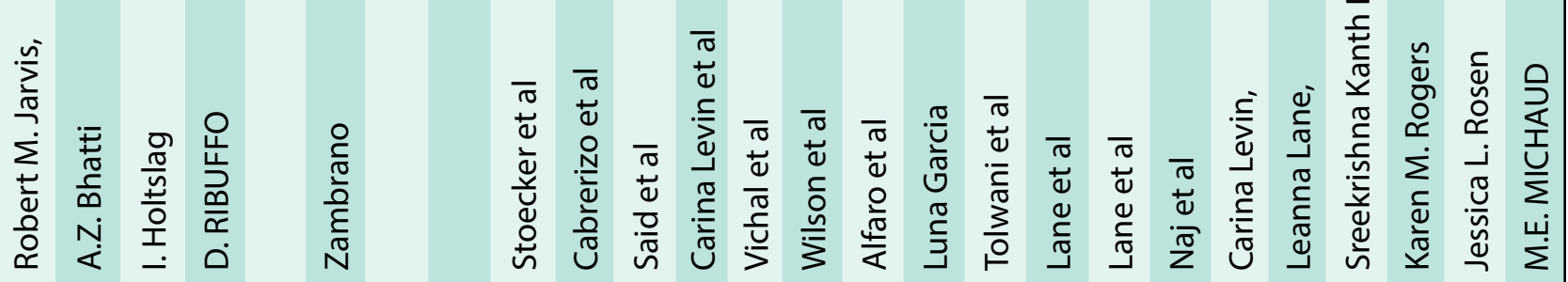
商

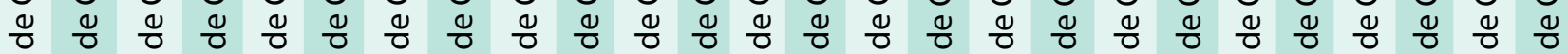

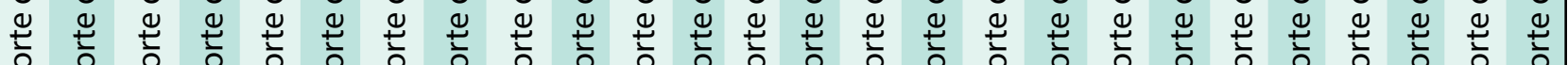

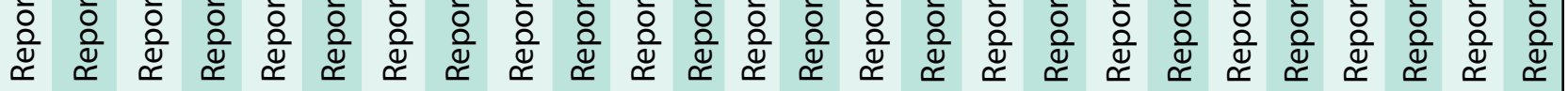


Tabla 3. Resultados del análisis de Regresión logística.

\begin{tabular}{|c|c|c|c|c|}
\hline & Coeficiente & OR & IC-95\% & P valor \\
\hline Sexo & 1,8 & 6,6 & $1,1-40,2$ & 0,039 \\
\hline Vómito & 2,3 & 10 & $1,8-55,6$ & 0,008 \\
\hline Fiebre & 3,1 & 21,7 & $2,2-215,7$ & 0,008 \\
\hline Hemoglobinuria & 3,9 & 52,6 & $2,9-928,1$ & 0,006 \\
\hline
\end{tabular}

Tabla 4. Protocolo de predicción del loxoscelismo sistémico.

\section{Puntaje}

Sexo masculino

2

Vómito

2

Fiebre

3

Hemoglobinuria

4

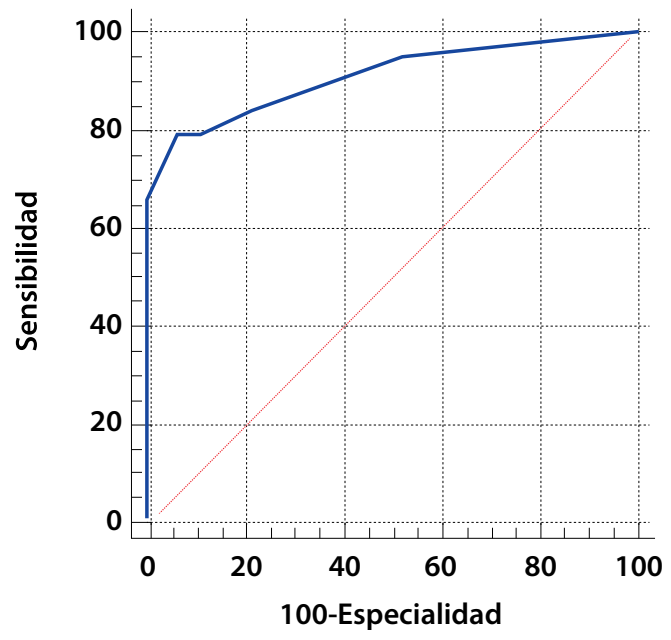

Figura 1. Curva ROC del protocolo de predicción clínica del loxoscelismo sistémico. Fuente: Del mismo autor.

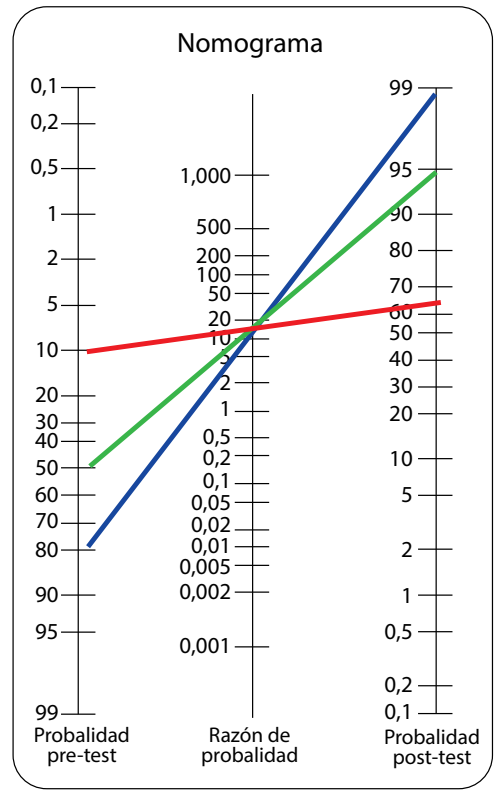

Figura 2. Nomograma de Fangan en la que se muestra tres escenarios clínicos simulados diferentes (Línea azul, roja y verde). Fuente: Del mismo autor. 


\section{DISCUSIÓN}

De acuerdo con el objetivo del estudio, se logró elaborar y validar un protocolo de predicción del compromiso sistémico en casos de loxoscelismo. Si bien las reglas de predicción clínica son una herramienta útil para guiar la toma de decisiones en dos escenarios clínicos específicos, como lo son el diagnóstico y el pronóstico de enfermedades y otros desenlaces clínicos, son solo una guía mas no un determinante en la toma de decisiones ${ }^{(6)}$. El protocolo de predicción del loxoscelismo derivado es el primero en su tipo y se espera que pueda ayudar a guiar las decisiones clínicas, tanto para mejorar la detección de casos hasta el ahorro de recursos en salud. Llama la atención que las variables incluidas dentro del modelo de predicción (vómito, fiebre, hemoglobinuria) son variables que han sido estudiadas y que han demostrado asociación estadística en investigaciones previas, como lo reportan los estudios de Webb et al y otros ${ }^{(4,7-9)}$; sin embargo, se incluye también a otra variable como el sexo masculino que no presenta investigaciones respecto a su asociación con el loxoscelismo sistémico. Por otro lado, Dahod et al ${ }^{(10)}$, determinó los predictores de falla renal aguda en mordedura de serpiente, encontrando que la hipotensión, albuminuria, tiempo de sangrado, tiempo de protrombina, hemoglobina, bilirrubina total y tiempo de mordedura hasta el ingreso al hospital predijeron adecuadamente el desarrollo de falla renal aguda. No obstante, en nuestro estudio estas variables no pudieron ser incluidas en el análisis estadístico por la presencia de la gran cantidad de datos perdidos, que podría ser producto de la gran variabilidad del manejo clínico por parte del personal médico. Es importante poder considerar en futuros estudios, la evaluación de estas variables en el loxoscelismo sistémico debido a que el cuadro producido por la mordedura de serpiente con efecto vásculo-tóxico es parecido al cuadro originado por el Loxosceles que también incluyen a la falla renal aguda.

La mediana de edad del grupo que presentó loxoscelismo sistémico fue significativamente diferente a la mediana de edad del grupo que no lo padeció. Webb et $\mathrm{al}^{(4)}$, reporta que el rango de edad de los pacientes con loxoscelismo sistémico se encuentra dentro de los 21 a 61 años y que no hay diferencias significativas entre los dos grupos. Por otro lado, Malaque et al ${ }^{(9)}$, también reportan que no hay diferencias entre los rangos de edad de los pacientes con loxoscelismo sistémico y cutáneo y en otro estudio realizado muestran que el rango de edad más frecuente es de 30 a 44 años. La edad juega un rol importante al momento del diagnóstico debido a que los pacientes que se encuentran en los grupos de edades extremas ( 2 años o 90 años), son los más propensos a desarrollar loxoscelismo sistémico, esta variable en nuestro estudio demostró ser una variable significativamente asociada, que no ha demostrado asociación en otros estudios. Sin embargo, en base a la variabilidad entre los reportes de casos y series de caso, deberían realizarse más estudios para evaluar su comportamiento en estudios posteriores.

El género más afectado por el loxoscelismo sistémico fue el masculino. Si bien el loxoscelismo es conocido por afectar con mayor frecuencia al género femenino, como lo reporta el informe de la Dirección General de Epidemiologia de la situación del loxoscelismo en Perú en el año $2013^{(11)}$, al igual que la frecuencia reportada por Nuñez et al ${ }^{(12)}$ en un estudio realizado en un hospital del Seguro Social EsSalud en Perú y por Valverde $^{(13)}$, en estudio realizado en el Hospital Regional

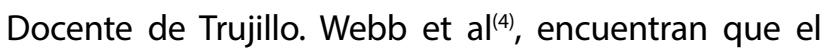
loxoscelismo sistémico afectó con mayor frecuencia a varones en comparación a las mujeres. Malaque et al(9), reporta que el género masculino predominó en los casos de loxoscelismo sistémico, en los cuales la bilirrubina indirecta era mayor a 7. Recomendamos que, al momento del abordaje clínico, una variable a considerar para el diagnóstico y manejo de un paciente con sospecha de loxoscelismo sistémico, sea el sexo masculino. Si bien en nuestro estudio, no fue incluida dentro del modelo de predicción por parte del análisis de regresión, es una variable clínicamente importante.

El tiempo de enfermedad mayor a 24 horas, fue el más frecuente en el grupo de loxoscelismo sistémico. Se conoce que es loxoscelismo sistémico, si un paciente con mordedura de araña se presenta dentro de las primeras 24 horas, siendo más probable que se trate de un loxoscelismo cutáneo al pasar este periodo de tiempo ${ }^{(14)}$. Una revisión actual realizada por Maguiña et $\mathrm{al}^{(15)}$, menciona que el tiempo para el desarrollo del loxoscelismo sistémico podría llegar hasta 36 horas posterior al accidente. Nuestros resultados afirman si es probable que se presente posterior a este periodo de tiempo, por lo que podemos concluir que independientemente del tiempo de evolución, el diagnóstico debería basarse en otras características del paciente que sumando nos deben dar una probabilidad aproximada del caso. Nuestro protocolo de predicción podría resolver esta brecha en el ámbito local.

Al momento del accidente, diferentes partes del cuerpo son afectadas, siendo más frecuente en las extremidades $^{(14)}$. Nuñez et $\mathrm{al}^{(12)}$, reporta que el lugar de presentación más frecuente del loxoscelismo fue en 
los miembros inferiores, siendo similar a los resultados encontrados en nuestra población de estudio. Por otro lado, hubo zonas del cuerpo, como la mordedura a nivel del abdomen que estuvieron asociadas significativamente a la presentación de loxoscelismo sistémico en compatibilidad a lo reportado por Webb et $\mathrm{al}^{(4)}$, quienes encuentran que una mordedura en abdomen, está asociado estadísticamente al desarrollo del loxoscelismo sistémico. Esta variable, pese a mostrar asociación, no fue incluida en el modelo de predicción, por lo que necesita una evaluación en una población más amplia a futuro, sin embargo, podría guiar nuestras decisiones clínicas. La mordedura en otras partes del cuerpo que no fuera el abdomen se asoció estadísticamente a una menor probabilidad de desarrollar loxoscelismo sistémico. Si bien la causa fisiopatológica no se conoce, posiblemente esta asociación sea debido a las características anatómicas y fisiológicas de las diferentes zonas del cuerpo. Es clínicamente importante considerar, en todo paciente con mordedura a nivel del abdomen, la posibilidad de desarrollar loxoscelismo sistémico.

Al evaluar la asociación estadística y la capacidad predictora de las diferentes variables estudiadas, la variable que presentó una mayor importancia por su fuerza de asociación y alta capacidad de predecir un loxoscelismo sistémico fue la hemoglobinuria. Se sabe que la hemoglobinuria, es producto de la hemólisis y la saturación de la haptoglobina, que lleva a la excreción elevada de la hemoglobina por parte de los riñones, observándose clínicamente como una orina oscura o hemoglobinuria causando daño renal debido a un taponamiento renal y que los cambios inflamatorios que produce en los túbulos renales pueden hacer evolucionar al paciente hacia una necrosis tubular aguda en muchos $\operatorname{casos}^{(16)}$. Si bien se conoce que la falla renal aguda, principal complicación y causa de mortalidad del loxoscelismo sistémico, es causada por el taponamiento renal, se ha demostrado que el veneno también presenta efectos directos sobre las células de los túbulos renales y que el sistema inmune cumple un papel fundamental dentro de la patología ${ }^{(16,17)}$. Sezerino et al ${ }^{(18)}$, reporta que solo el $28,6 \%$ de los pacientes con loxoscelismo sistémico presenta hemoglobinuria. Webb et al(4), reportan una cifra diferente, llegando a presentar hemoglobinuria hasta en un $48 \%$ los pacientes con loxoscelismo sistémico. Alva-Medina et al ${ }^{(19)}$, reportan una frecuencia de hemoglobinuria en niños con loxoscelismo sistémico del 61,1\% en Perú. Al comparar estas evidencias con nuestros resultados, en efecto, la hemoglobinuria es una variable importante a considerar al momento del diagnóstico del loxoscelismo sistémico, demostrando su alto poder predictivo en nuestros hallazgos. Sin embargo, puede no presentarse en todos los casos, por tal motivo el modelo de predicción clínica debe ser validado en una población más amplia.

La fiebre es un síntoma asociado al loxoscelismo sistémico y podría alertanos de la presencia de un loxoscelismo sistémico en un paciente con mordedura de araña ${ }^{(15)}$. Además, la fiebre se asocia a la presencia de hemólisisi(9). Sin embargo, Malaque et $\mathrm{al}^{(20)}$, reporta que solo el $16 \%$ de los pacientes con loxoscelismo presenta un bajo grado de fiebre. En otro estudio realizado por el mismo autor y colaboradores encuentran lo contrario, que la fiebre se presenta en el $68 \%$ de los pacientes con sospecha de loxoscelismo sistémico ${ }^{(9)}$. La capacidad predictora de la fiebre ha mostrado un importante papel en nuestro protocolo, siendo importante su consideración al momento del abordaje de un paciente con sospecha de loxoscelismo sistémico. El vómito es otra variable importante a considerar en el diagnóstico, y es descrita como un síntoma que puede ser observado en el loxoscelismo sistémico ${ }^{(15,14)}$. Esta variable, junto a las anteriores, es clínicamente relevante para el diagnóstico, como resultado obtenemos una mejora en el manejo del loxoscelismo sistémico y una reducción de gastos de los recursos de salud. Podemos también predecir, cuándo un paciente puede desarrollar o no un loxoscelismo sistémico posterior a una mordedura, que sería valor agregado de nuestro modelo de predicción.

La mortalidad en ambos grupos fue baja. En el grupo de loxoscelismo cutáneo, el paciente falleció por una infección asociada al cuadro cutáneo. En este aspecto toma importancia el manejo clínico del loxoscelismo sistémico, el cual es principalmente de soporte ${ }^{(15)}$. Un manejo adecuado determina la buena o mala evolución del paciente e influye directamente sobre la mortalidad. En este sentido, al parecer el manejo de estos casos fue adecuado y por lo tanto la mortalidad fue baja en el loxoscelismo sistémico.

La primera limitación es el número reducido del tamaño de muestra, que afecta directamente al poder estadístico y generalización de los resultados a poblaciones más grandes, sin embargo, la diversidad de los casos incluidos pertenecientes a diferentes partes del mundo hace aplicable el modelo también a poblaciones que se encuentran tanto dentro como fuera del país, convirtiendo a la metodología utilizada en un aporte para el estudio de enfermedades raras. La segunda limitación, es la importante cantidad de datos perdidos, que condicionaron la eliminación a una parte considerable de las variables operacionalizadas para el análisis estadístico, sin embargo, que no 
fueron determinantes para el desarrollo de la presente investigación ni para la derivación del modelo de predicción, no obstante, limitaron la inclusión de otras variables. Motivo por el que recomendamos replicar el presenteestudioenunapoblaciónampliaymulticéntrica para garantizar el poder muestral, resultados robustos, inclusión de otras variables y mayor generalización de los resultados, y beneficiar a poblaciones que no compartan las mismas características de la población estudiada.

La regla de predicción clínica del loxoscelismo sistémico derivado es válida y aplicable a poblaciones que se encuentran dentro y fuera del país. La metodología utilizada es un aporte para el estudio de enfermedades raras. Se requiere una validación externa en una amplia población: evaluación en condiciones clínicas reales e inclusión de variables adicionales.

\section{REFERENCIAS BIBLIOGRÁFICAS}

1. Akdeniz S, Green JA, Stoecker WV, Gomez HF, Keklikçi SU. Diagnosis of loxoscelism in two Turkish patients confirmed with an enzyme-linked immunosorbent assay (ELISA) and non-invasive tissue sampling. Dermato Online J. 2007;13(2):11. Disponible en: https://www.ncbi.nlm.nih.gov/ pubmed/17498430

2. Stoecker WV, Wasserman GS, Calcara DA, Green JA, Larkin K. Systemic loxoscelism confirmation by bite-site skin surface: ELISA. Mo Med. 2009;106(6):425-7,431. Disponible en: https://www.ncbi.nlm.nih.gov/pmc/ articles/PMC3160791/

3. Gomez HF, Krywko DM, Stoecker WV. A new assay for the detection of Loxosceles species (brown recluse) spider venom. Ann Emerg Med. 2002;39(5):469-74. https://doi.org/10.1067/mem.2002.122914

4. Webb C, María C, Maguiña C, González E. Factores asociados a la presentación víscero-hemolítica de loxoscelismo en el Hospital Nacional Cayetano Heredia, entre el 2000 y 2008. Acta Médica Peruana. 2010;27(1):2936. Disponible en: http://www.scielo.org.pe/scielo.php?script=sci arttext\&pid=S1728-59172010000100006\&lng=es\&nrm=iso\&tlng=es

5. Han X, Duan M, Hu L, Zhou D, Zhang W. Plasmablastic lymphoma Medicine (Baltimore) [Internet]. 2017 [citado 28 Mar 2017];96(9). https:// dx.doi.org/10.1097\%2FMD.0000000000005981

6. Collins GS, Reitsma JB, Altman DG, Moons KGM. Transparent Reporting of a multivariable prediction model for Individual Prognosis or Diagnosis (TRIPOD): the TRIPOD statement. Ann Intern Med. 2015;162(1):55-63. https://doi.org/10.7326/M14-0698

7. Harz-Fresno I, Manterola P, Ruiz M, Abud C. Loxoscelismo cutáneo visceral: actualización en el manejo a propósito de un caso. Revista chilena de infectología. 2015;32(2):230-3. http://dx.doi.org/10.4067/S071610182015000300014

Quispe AJ. Loxoscelismo en adultos morbi-mortalidad y factores asociados al desarrollo de la forma viscero - hemolitica [Tesis para optar el Título Profesional de Médico Cirujano]. [Arequipa]: Universidad Nacional de San Agustin; 1997

7. Malaque CMS, Santoro ML, Cardoso JLC, Conde MR, Novaes CTG, Risk $J Y$, et al. Clinical picture and laboratorial evaluation in human loxoscelism Toxicon. 2011;58(8):664-71. https://doi.org/10.1016/j.toxicon.2011.09.011

9. Dharod MV, Patil TB, Deshpande AS, Gulhane RV, Patil MB, Bansod YV. Clinical Predictors of Acute Kidney Injury Following Snake Bite Envenomation. N Am J Med Sci. 2013;5(10):594-9. https://dx.doi. org/10.4103\%2F1947-2714.120795
Agradecimiento: Al Dr. Alonso Soto-Tarazona, por el apoyo brindado en la concepción de la idea y diseño del estudio.

Contribuciones de autoría: Los autores participaron en la génesis de la idea, diseño de proyecto, recolección e interpretación de datos, análisis de resultados y preparación del manuscrito del presente trabajo de investigación.

\section{Financiamiento: Autofinanciado.}

Conflicto de interés: Los autores declaran no tener conflicto de interés en la publicación de este artículo.

Recibido: 07 de noviembre del 2019

Aprobado: 30 de diciembre del 2019

Correspondencia: Rafael Pichardo-Rodriguez.

Dirección: Av. Javier Prado Este 3028, San Borja 15037, Lima - Perú.

Teléfono: 986332210

Correo:rafael_martin1352@hotmail.com
10. Vargas E. Situación del loxoscelismo en el Perú. 2013. Bol Epidemiol. 2013;23(5):74-5. Disponible en: http://www.dge.gob.pe/portal/docs/ vigilancia/boletines/2014/05.pdf

11. Núñez-Moscoso L, Chacón-Arévalo S. Aspectos clínicos yepidemiológicos del loxoscelismo en un hospital de primer nivel. Rev Soc Peru Med Interna. 2013;26(1):14-6. Disponible en: http://www.medicinainterna.org.pe/pdf/ trabajo\%20original\%203.pdf

12. Valverde J. Aspectos clínicos y epidemiológicos del loxocelismo, Hospital Regional Docente de Trujillo, enero 2001 a noviembre 2003. Folia Dermatol. 2003;14(2):15-9. Disponible en: http://sisbib.unmsm.edu.pe/bvrevistas/ folia/Vol14_N3/trab2.htm

13. Mendoza-Ticona C, Cabezas-Sánchez C. Loxoscelismo: Evaluación Clínica, Tratamiento y Prevención. Revista SPEIT. 2006;1:2-8. Disponible: http://sisbib.unmsm.edu.pe/bvrevistas/speit/2006_n1/pdf/a02.pdf

14. Vargas CM, Vásquez VF, Ugarte RP. Actualización sobre manejo de araneismo en Perú. Revista Medica Herediana. 2017;28(3):200. https://doi. org/10.20453/rmh.v28i3.3189

15. Anwar S, Torosyan R, Ginsberg C, Liapis H, Morrison AR Clinicopathological course of acute kidney injury following brown recluse (Loxoscles reclusa) envenomation. Clin Kidney J. 2013;6(6):609-12. https:// dx.doi.org/10.1093\%2Fckj\%2Fsft 111

16. Nag A, Datta J, Das A, Agarwal AK, Sinha D, Mondal $S$, et al. Acute kidney injury and dermonecrosis after Loxosceles reclusa envenomation. Indian J Nephrol. 2014;24(4):246-8. https://dx.doi. org/10.4103\%2F0971-4065.133017

17. Sezerino UM, Zannin M, Coelho LK, Gonçalves Júnior J, Grando M, Mattosinho SG, et al. A clinical and epidemiological study of Loxosceles spider envenoming in Santa Catarina, Brazil. Trans R Soc Trop Med Hyg. 1998;92(5):546-8. https://doi.org/10.1016/S0035-9203(98)90909-9

18. Medina A, Jhosep D. Loxoscelismo En Niños: Epidemiología, Aspectos Clínicos Y Tratamiento. Hospital Belén De Trujillo, 1990 - 2010. Universidad Nacional de Trujillo [Internet]. 2013 [Citado 7 Ener 2018]; Disponible en: http://dspace.unitru.edu.pe/handle/UNITRU/624

19. Málaque CMS, Castro-Valencia JE, Cardoso JLC, França FO de S, Barbaro KC, Hui WF. Clinical and epidemiological features of definitive and presumed loxoscelism in São Paulo, Brazil. Revista do Instituto de Medicina Tropical de São Paulo. 2002;44(3):139-43. http://dx.doi.org/10.1590/S003646652002000300005 